\title{
Patrones de consumo de alcohol en adolescentes ecuatorianos: ¿Estilo mediterráneo o anglosajón?
}

\author{
Rodrigo Moreta-Herrera1, Marlon Mayorga-Lascano², Lorena León-Tamayo ${ }^{3}$ y Tábata \\ Naranjo-Reyes ${ }^{4}$
}

\begin{abstract}
RESUMEN
Introducción: Se conoce una práctica del consumo generalizada de alcohol en adolescentes, aunque no el patrón o tipo de consumo más representativo. Objetivo: Identificar por sexo los niveles y los patrones de consumo de alcohol en una muestra de adolescentes ecuatorianos. Metodología: Estudio descriptivo, categórico y comparativo a través del uso de la prueba AUDIT. Participantes: 1260 adolescentes (55,3\% hombres), de 12 a 19 años $(M=15 ; D E=1,56)$, estudiantes de 11 centros (86\% públicos), en ciclo de Educación Básica General (30.2\%) y Bachillerato (69.8\%) de las provincias de Cotopaxi y Tungurahua. Resultados: El consumo de alcohol se encuentra extendido en la muestra, la abstinencia es del $43 \%$ y el $49 \%$ en hombres y mujeres, el tipo de consumo no presenta riesgos significativos. El patrón de consumo de alcohol es mayoritariamente moderado; la prevalencia del estilo intenso es del 15.7 y el $22.2 \%$ en hombres y mujeres. El sexo es invariante. Conclusiones: Hay una práctica generalizada de consumo de alcohol moderado y un patrón de consumo del tipo mediterráneo.
\end{abstract}

Palabras clave: adolescencia, alcohol, nivel, sexo, patrón.

\section{Alcohol use patterns predominate in ecuatorians adolescents: Mediterranean or Anglo-Saxon style?}

\begin{abstract}
Introduction: A practice of generalized alcohol consumption in adolescents is known, although not the most representative pattern or type of consumption. Objective: Identify by gender, the levels and patterns of alcohol use in a sample of Ecuadorian adolescents. Methodology: Descriptive, categorical and comparative study with the AUDIT. Sample: 1260 adolescents (55.3\% men), 12 to 19 years old $(M=15 ; S D=1.56)$, students from 11 centers (86\% public), in the General Basic Education cycle (30.2\%) and High School $(69.8 \%)$ of Cotopaxi and Tungurahua provinces. Results: Alcohol use is widespread in the sample, abstinence is $43 \%$ and $49 \%$ in men and women, the type of alcohol use does not present significant risks and the alcohol use pattern is low and moderate. The pattern of
\end{abstract}

${ }^{1}$ Pontificia Universidad Católica del Ecuador, Ecuador; rmoreta@pucesa.edu.ec; https://orcid.org/00000003-0134-5927

2 Pontificia Universidad Católica del Ecuador, Ecuador;pmayorga@pucesa.edu.ec; https://orcid.org/00000002-2515-4159

3 Pontificia Universidad Católica del Ecuador, Ecuador; aleon@pucesa.edu.ec; https://orcid.org/00000001-8151-7893

4 Pontificia Universidad Católica del Ecuador, Ecuador; tabata.r.naranjo.r@pucesa.edu.ec; https://orcid.org/0000-0002-5550-8544

AGRADECIMIENTOS | Los autores agradecemos a la Pontificia Universidad Católica del Ecuador Sede Ambato por la financiación al proyecto intitulado "Procesos cognitivos razonados y automáticos en la valoración del alcohol y el consumo en adolescentes de la ciudad de Ambato" como parte de la VII Convocatoria de Proyectos de Investigación del año 2017 y fruto de este se reflejan los presentes resultados. 
alcohol use is mostly moderate; the intense style prevalence is $15.7 \%$ and $22.2 \%$ in men and women. The gender is invariant. Conclusions: There is a widespread practice of moderate alcohol use and a pattern of Mediterranean-type alcohol use.

Keywords: adolescents, alcohol, level, sex, patterns.

El alcohol es la sustancia psicoactiva con más consumo entre adolescentes y jóvenes (Wilsnack, Wilsnack, \& Kantor, 2013; Gaete et al., 2016; Moreta-Herrera, Ilaja-Verdesoto, Mayorga-Lascano, León-Tamayo, \& López-Castro, 2018a). Su consumo, junto con el de tabaco, representa un problema de salud pública mundial (World Health Organization, 2013), y tiene una incidencia marcada en los costes de los sistemas nacionales de salud, al punto que representa, al menos, el $5 \%$ de la carga global de enfermedades (Rehm et al., 2009).

En la adolescencia, la abstinencia al consumo es cada vez menos frecuente (World Health Organization, 2014; Vieno, Altoè, Kuntsche, \& Elgar, 2018). La edad de inicio del consumo se acorta cada año y la media roza los 12 años (Center for Behavioral Health Statistics and Quality, 2018; Observatorio Español de las drogas y las adicciones, 2018). Este inicio precoz incide en la relación del consumo de alcohol con los efectos negativos en edades futuras a corto y mediano plazo (juventud y adultez) (MaldonadoMolina, Reingle, \& Jennings,2011; Kunstsche et al., 2015; Liang \& Chikritzhs, 2015; Miller et al., 2016). Pues la consolidación del consumo y la combinación con otras sustancias también se dará de forma más temprana (entre los 15 a 16 años) (Zurita \& Álvaro, 2014).

América y Europa son las regiones con el mayor número de usuarios de alcohol (53\% y 70\%, respectivamente, en adolescentes) (World Health Organization, 2014). Por citar algunos ejemplos, en Estados Unidos la prevalencia fluctúa entre el $47.5 \%$ y el $57.1 \%$ para mujeres y hombres adolescentes (SAMHSA, 2014). En España es del $55.1 \%$ (12\% reportan consumo alto y 42\% consumo medio) (Zurita \& Álvaro, 2014). En México, incurren en el consumo el $46 \%$ y el $54 \%$ de hombres y mujeres (ArriojaMorales, Frari Galera, Gargantúa Aguila, de Ávila Arroyo, \& Morales Castillo, 2017); En Chile, el 35,6\% reporta consumo durante el último mes y el 63\% durante el último año (Servicio Nacional para la Prevención y Rehabilitación del consumo de drogas y alcohol, 2013). Mientras que en Argentina, el 32,3\% refiere consumo actual bajo y moderado y un $17.2 \%$ con episodios de consumo excesivo (Morello et al., 2017)

En el caso del Ecuador, los reportes señalan que el consumo entre adolescentes es relativamente bajo en intensidad y frecuencia (MayorgaLascano, Moreta-Herrera, León-Tamayo, \& Troncozo-Guevara, 2019). La incidencia del consumo a nivel nacional es del 31\% (Consejo Nacional de Control de Sustancias Estupefacientes y Psicotrópicas, Observatorio Nacional de Drogas, 2013). En otros estudios se revela que el consumo durante el último mes es del $22.5 \%$, con mayor consumo en hombres que en mujeres (Moreta-Herrera et al., 2018a). También, que un $23.7 \%$ de adolescentes ya presentan un consumo de alcohol riesgoso (según categorización del AUDIT) (Paqui Baho, 2017). Y quienes ya presentan un consumo más frecuente de 
alcohol, presentan riesgo psicosocial en un 91.3\% (Ramón Durán \& Dávila Pontón, 2018).

La incidencia del consumo de alcohol responde a una compleja cuestión multifactorial atribuida a factores de riesgo asociados a aspectos biológicos, psicológicos, familiares, sociales, económicos, entre otros. Con lo cual se expone una idea de lo complejo que es este fenómeno, en especial entre los adolescentes. Estos factores incluyen la edad del consumidor y la edad de inicio (Liang \& Chikritzhs, 2015), los problemas conductuales, el consumo de padres y figuras cercanas (Gaete et al., 2016), el ambiente familiar, los niveles deficientes de madurez cognitiva y emocional (Zurita \& Álvaro, 2014), la influencia, la presión social y la percepción de bajo riesgo sobre el consumo de los pares (Pérez \& Lara, 2008; Wesche, Kreager, \& Lefkowitz, 2018) y la exposición a información sobre el consumo en redes sociales (Navarro-Oliva Robledo, Medina, Candía-Arredondo, \& Facundo, 2016). A más de ello, también se encuentra el contexto académico (Moreta-Herrera, MayorgaLascano, León-Tamayo, \& Ilaja-Verdesoto, 2018b) y la vulnerabilidad socioeconómica de la familia (Cerdá, Diez-Roux, Tchetgen Tchetgen, GordonLarsen, \& Kiefe, 2010).

Un aspecto relevante como factor de riesgo corresponde al sexo. Varios estudios recogen que el consumo de alcohol es mayor en los hombres que en las mujeres (World Health Organization, 2014; Miller et al., 2016; ArriojaMorales et al., 2017; Moreta-Herrera et al., 2018a), que se evidencia también en las consecuencias a nivel físico y psicosocial (Schulte, Ramo, \& Brown, 2009). Sin embargo, el crecimiento del consumo de las mujeres señala aumentos significativos y existe, al parecer, cada vez más, una reducción de la brecha de consumo entre hombres y mujeres (Wilsnack, Wilsnack, \& Kantor, 2013).

\section{Patrones de consumo de alcohol}

El consumo de alcohol es una práctica común entre los adolescentes. La existencia de un consumo único y/o esporádico no es un referente significativo de peligrosidad, sino su consolidación y, en especial, la pérdida del control del consumo mismo (Gea et al., 2014). Para establecer patrones es importante considerar la cantidad que se bebe, la cual se valora por el número de unidades de bebidas estándar (UBE) o trago estándar (TE) (World Health Organization, 1994), que es la cantidad en gramos de alcohol (entre 8-9 gramos) ingeridos, independientemente del tipo de bebida (cerveza, vino, ron, otros). Esto, para establecer equivalentes aproximados entre bebidas de consumo. Así, por ejemplo, un vaso de cerveza de $5 \%$ grados de alcohol es similar al de una copa de vida de $12 \%$ y al de un trago de una bebida espirituosa (whisky, ron, vodka y similares) de 40\%.

La habituación del consumo o el patrón en sí de los adolescentes está sujeto a distintos factores. Sin embargo, el aspecto cultural es altamente relevante. Ya se había considerado que uno de los principales es la cantidad de alcohol que se consume expresado en UBE, pero también es importante considerar la frecuencia, así como el contexto. En este sentido, las formas de clasificar los patrones de consumo, tradicionalmente se realizan en función del consumo per cápita que tienen los bebedores. De esta manera se clasifican entre culturas mojadas o wet cultures y secas o dry cultures (Galán, González, $\&$ Valencia-Martín, 2014), clasificación que en muchos aspectos toma como 
referencia los países europeos en los que el consumo ha adquirido ciertas especificaciones.

Por una parte, el consumo moderado, 'mediterráneo' o dry (Heath, 1997; Strangers et al., 2006), se basa en un consumo mesurado de bebidas de baja concentración (vino y cerveza), con acceso poco restrictivo, complementario con la alimentación, con familiaridad e integrado en la cotidianidad diaria. Mientras que el consumo intenso, binge drinking, wet o 'anglosajón' (ValenciaMartín, Galán, \& Rodríguez-Artalejo, 2007), comprende un consumo agudo de bebidas con alta concentración (suele combinarse), de alta restricción social, sin el acompañamiento de alimentos y sin formar parte de la cotidianidad diaria (fines de semana). En el primer patrón, es característico que la tasa de abstinencia sea modestamente baja y su inicio en edades tempranas, mientras que en el segundo es frecuente la intoxicación con un inicio relativamente más tardio (Galán et al., 2014). Y aunque el consumo intenso implica más riesgo, el consumo moderado también guarda relación con las consecuencias en los trastornos por consumo de alcohol (Wadolowski et al., 2015), la morbilidad y la mortalidad (Gea et al., 2014).

Al profundizar en el consumo intenso, este patrón se presenta con episodios de consumo intenso de alcohol o heavy episodic drink (ECIA). Cada episodio se conforma por la ingesta de 5 o más UBE en un tiempo corto (Golpe, Isorna, Barreiro, Braña, \& Rial, 2017) y en condición de riesgo se establece al menos un ECIA como mínimo una vez durante dos semanas. En la adolescencia, los ECIA son más frecuentes, aunque con diferencias por sexo en la etapa media (de 14 a 15 años) y tardía (de 17 a 18 años) que en la adolescencia inicial (de 12 y 13 años) (Miller et al., 2016). La información epidemiológica de los ECIA es limitada y no cuenta con una sistematización amplia. Como marco referencial podemos aportar, a partir de estudios en España, prevalencias del 16,5\% durante el último mes y el 34.4\% durante el último año (adolescentes de 12 a 18 años) (Golpe et al., 2017). En México, con el $44 \%$ y el $21 \%$ para el último año y mes, respectivamente, en hombres y para las mujeres del 25.9\% y el 8.6\% (Villarreal-González, Sánchez-Sosa, \& Musitu Ochoa, 2013). En el Ecuador, es del 9.7\% y con un 10\% que reportan consumo al menos una vez al mes (Ramón Durán \& Dávila Pontón, 2018).

\section{Importancia y dificultades metodológicas en el estudio de las conductas de consumo}

La importancia científica del estudio de los patrones de consumo, en especial de los ECIA, se debe a que estos ayudan a determinan el uso posterior del alcohol y la dependencia (Gmel, Kuntsche, \& Rehm, 2011); y a las repercusiones a nivel físico, psicológico y social a lo largo de la vida (LeónMuñoz, Guallar-Castillón, García-Esquinas, Galán, \& Rodríguez-Artalejo, 2017). En este sentido, es necesario considerar algunos aspectos metodológicos que dificultan generalmente estos estudios.

Uno de ellos se relaciona con el reporte de las cifras. Pues son diversas y con variadas metodologias. Especialmente por las definiciones operativas que reciben los constructos asociados al consumo (contacto, consumo moderado, riesgoso, binge drink, hard drink, otros) (Galán et al., 2014), así como por la medición del consumo de alcohol propiamente dicho que, si bien es cierto se lo hace en UBE, la cantidad de grados que se consideran para un UBE puede variar entre distintos países (World Health Organization, 1994). Esto dificulta la estandarización y el intercambio de conocimientos y limita la tipificación de 
la condición del consumo y la categorización de los consumidores adecuadamente (Golpe et al., 2017).

Si bien es cierto que muchas de estas definiciones se realizan a partir de observaciones realizadas en poblaciones de países europeos y anglosajones, en especial para definir conceptos como 'patrón de consumo' (Heath, 1997; Strangers et al., 2006; Valencia-Martín et al. 2007); estos modelos se toman erróneamente como referenciales para 'etiquetar' a otras poblaciones, perdiendo el sentido de la exploración y la búsqueda de soluciones prácticas. Sin embargo, se debe considerar que los estudios en sí mismos sobre patrones de conducta son limitados y con pruebas específicas de medición en debate.

También se deben considerar las limitaciones metodológicas a nivel de instrumentos para medir la conducta de consumo. Por ejemplo, se presenta el caso del AUDIT (Saunders, Aasland, Babor, De la Fuente, \& Grant, 1993), el cual se organiza en función de los criterios del Trastorno por Consumo de Alcohol, pero no tiene un consenso total sobre los adecuados puntos de corte para el screaning en adolescentes, así como para la diferenciación por sexo (Boubeta, Ferreiro, Gallego, Tobío, \& Mallou, 2017). Por lo expuesto, estos aspectos corren el riesgo de arrojar error o sesgo.

Finalmente, una limitación más corresponde a la condición de los estudios sistematizados sobre la práctica del consumo en la región americana, en especial en el Ecuador, pues se disponen de pocos datos consolidados y continuos que revelen la dinámica del consumo. En este caso, el avance de investigación es de reciente desarrollo.

\section{Objetivos e hipótesis}

Tras la revisión planteada, se presentan como objetivos de este estudio: a) identificar los niveles y los patrones de consumo de alcohol dominantes en una muestra de adolescentes del Ecuador, y b) conocer las diferencias de sexo existentes. Las hipótesis planteadas consideradas por lo tanto son: a) El nivel de consumo de alcohol es bajo entre los adolescentes, b) el patrón de consumo característico de la población es el mediterráneo o moderado y c) el consumo en los hombres es significativamente mayor al registrado en las mujeres.

\section{METODOLOGÍA}

\section{Diseño}

El presente trabajo es un estudio de carácter descriptivo, comparativo por sexo, de corte transversal (Ato, López, \& Benavente, 2013). Además, se muestra la categorización diagnóstica que representa la condición de consumo de alcohol, los patrones de consumo y las diferencias por sexo.

\section{Participantes}

La muestra del estudio está constituida por 1260 participantes (55.3\% hombres). Las edades rondan entre los 12 a los 19 años $(M=15 ; D E=1.56)$. E1 93.3\% de la muestra se identifica como mestizos/as. Los participantes son de las provincias de Cotopaxi (61.8\%), de las ciudades de Latacunga (30.1\%) y Salcedo (31.7\%), y de Tungurahua (38.2\%), de las ciudades de Ambato (25.9\%) y Pelileo (12.3\%), en el Ecuador. Están ubicados en el sector urbano el $69.4 \%$ y en el rural el 30.6\%. El 24.4\% reporta criterios de vulnerabilidad económica. 
Son estudiantes de 11 centros educativos (86\% públicos), que cursan entre el ciclo de Educación General Básica [EGB] (30.2\%), de 8vo a 10mo año, y Bachillerato $(69.8 \%)$ de 1 ro a 3 er año. El $15.8 \%$ reporta un rendimiento académico bajo, mientras que el $12.1 \%$ presenta pérdida de año y un $6.6 \%$ indica que combinan sus estudios con actividades laborales.

La selección de los participantes se realizó a través de un muestreo de tipo no probabilístico con criterios de inclusión. Estos son a) Participación voluntaria, b) Autorización escrita expresa del representante o tutor legal, c) ser estudiante matriculado en uno de los centros del presente estudio, y d) Asistencia regular a clases.

\section{Instrumentos}

Cuestionario para la identificación de los trastornos debido al consumo de alcohol (AUDIT; World Health Organization, 1992; Saunders et al., 1993) en la versión adaptada al castellano (Rubio, Bermejo, Cabellero, \& Santo Domingo, 1998). Este instrumento busca valorar por medio de diez preguntas el Consumo de riesgo de alcohol (CRA), los Sintomas de dependencia (SDA) y el Consumo perjudicial (CPA) por medio de una escala Likert de cinco niveles. E1 AUDIT cuenta con niveles aceptables de fiabilidad en adolescentes con $a=.82$ (Boubeta et al., 2017), así como dentro del contexto latinoamericano y español con valores de .93 (de la Villa Moral Jiménez, Bringas Molleda, Ovejero Bernal, Morales Quintero, \& Rodríguez Díaz, 2017). La fiabilidad encontrada para este trabajo fue de $a=.84$, equivalente a aceptable.

El AUDIT establece dos categorias diagnósticas: Bebedor de riesgo (8 a 14 puntos) y Problemas físicos y psíquicos (probable dependencia) con 15 puntos o más). Las puntuaciones en cero reflejan abstención total al alcohol y los puntajes de 1 a 7 implican un consumo no perjudicial.

Por otra parte, el consumo intenso de alcohol o binge drinking se puede identificar analizando la pregunta dos y tres. El primero, para los Episodios de Consumo Intenso de Alcohol (ECIA), al analizar la pregunta 2 (más de cinco ingestas de UBE un día típico de consumo) y la pregunta 3 (que los ECIA se den al menos una vez cada dos semanas) (Golpe et al., 2017).

\section{Procedimiento}

El desarrollo de este reporte contó con la identificación de los centros educativos participantes y la autorización de estos para su desarrollo. Se seleccionó a los voluntarios tras un proceso de socialización del estudio en el que se expusieron los objetivos del estudio y los requisitos de participación, que incluía la autorización por medio del consentimiento por escrito de los tutores legales. El proceso de evaluación se realizó en los centros educativos y fueron aplicados de manera grupal con un tiempo aproximado de 35 minutos. Posteriormente, se depuró y digitalizó la información y se realizaron los análisis estadísticos respectivos para la verificación de las hipótesis propuestas. La presentación de los reportes investigativos contó con la aprobación de la Comisión de Publicaciones de la Pontificia Universidad Católica del Ecuador, Sede Ambato, que es la encargada de revisar procesos y protocolos éticos para publicaciones.

\section{Análisis de datos}

El tratamiento estadístico comprende en una primera parte un análisis descriptivo comparativo por sexo de los puntajes medios alcanzados en los 
componentes constitutivos del AUDIT, así como la valoración global. Para la identificación de diferencias estadísticas $(p<.05)$ se aplicó la prueba $t$ para muestras independientes ( $t$ ) y el tamaño del efecto con la prueba de Hedges ajustada $\left(g_{a j}\right)$. El segundo bloque lo constituyen los análisis comparados por sexo sobre la base de las Categorias Diagnósticas, la Frecuencia del Consumo, el Patrón de Consumo y la frecuencia de los ECIA. El contraste es con la prueba Chi cuadrado de Pearson $\left(x^{2}\right)$. También se incluye un análisis por edad de la media del consumo de alcohol entre los participantes del estudio. La gestión informática de los resultados se llevó a cabo gracias al uso del software $R$ Commander (R Core Team, 2015), el cual es de libre disponibilidad.

\section{RESULTADOS}

\section{Análisis comparativo por sexo del consumo de alcohol}

En la tabla 1, se observa que la media del AUDIT reporta 3,8 puntos, los niveles de consumo de alcohol y de consecuencias son bajos. Al encontrarse la media del consumo por debajo del punto de corte ( $p \leq 7$ puntos), se establece que el consumo no representa riesgo, en gran parte debido a que el mismo se caracteriza por una baja ingesta de alcohol y una frecuencia esporádica. Al analizar diferencias, tanto en hombres como en mujeres, no se presentan diferencias por sexo, por lo que los grupos pueden considerarse iguales.

Tabla 1.

Análisis comparativo por sexo del consumo de alcohol (AUDIT)

\begin{tabular}{|c|c|c|c|c|c|c|c|}
\hline \multirow{2}{*}{ FACTORES } & \multicolumn{2}{|c|}{ Hombres } & \multicolumn{2}{|c|}{ Mujeres } & \multirow{2}{*}{$F$} & \multirow{2}{*}{$t_{(1258)}(p)$} & \multirow{2}{*}{$\boldsymbol{g}_{(a j .)}$} \\
\hline & $M$ & $D E$ & $M$ & $D E$ & & & \\
\hline Consumo Riesgoso & 1.8 & 2.1 & 1.6 & 2.2 & 0.009 & $1.2(.212)$ & .09 \\
\hline Síntomas de dependencia & 0.8 & 1.4 & 0.7 & 1.5 & 0.011 & $0.5(.617)$ & .07 \\
\hline Consumo Perjudicial & 1.4 & 2.3 & 1.3 & 2.2 & 0.609 & $0.9(.387)$ & .04 \\
\hline AUDIT & 3.9 & 4.9 & 3.6 & 5.1 & 0.020 & $1.1(.284)$ & .06 \\
\hline
\end{tabular}

\section{Categorías diagnósticas del consumo de alcohol y abstinencia}

Como se puede observar en la tabla 2, según tipifica el AUDIT, los participantes se categorizan tres grupos diferenciados. El mayor grupo, que bordea el 76\% (AUDIT < 8 puntos) es el que presenta un nivel de consumo y de exposición al daño bajo y no representa un riesgo significativo; seguido por el 22\% (AUDIT entre 8 a 15 puntos), cuyo consumo se presenta riesgoso y con dificultades asociados al consumo de alcohol. $Y$ un grupo del 1,8\%, aproximadamente, cuyos valores (AUDIT > 15 puntos) reflejan problemas físicos y psicosociales y una probable dependencia asociada al consumo de alcohol. No se presentan diferencias significativas $(p>.05)$ por sexo, por lo que se considera que los niveles de consumo y abstinencia son similares entre los grupos. 
Tabla 2.

Análisis comparativo por sexo de las categorías diagnósticas del AUDIT

\begin{tabular}{lccccccc}
\hline \multirow{2}{*}{ VARIABLES } & \multicolumn{2}{c}{ Total } & \multicolumn{2}{c}{ Hombres } & \multicolumn{2}{c}{ Mujeres } & Contraste \\
\cline { 2 - 8 } & $n$ & $\%$ & $n$ & $\%$ & $n$ & $\%$ & $X^{2}(p)$ \\
\hline Sin riesgo & 958 & $76.2 \%$ & 522 & $74.9 \%$ & 436 & $77.4 \%$ & \\
Consumo de Riesgo & 280 & $22.0 \%$ & 163 & $23.4 \%$ & 117 & $20.8 \%$ & $1.22(.543)$ \\
Problemas por consumo & 22 & $1.8 \%$ & 12 & $1.7 \%$ & 10 & $1.8 \%$ & \\
\hline
\end{tabular}

Nota: Total: 1260 casos; Hombres: 697; Mujeres: 563; $x^{2}$ : Chi cuadrado de Pearson; $p$ : significancia

\section{Patrones de consumo de alcohol y episodios de consumo intenso}

La tabla 3 muestra que el nivel de abstinencia entre los participantes (Pregunta 1 AUDIT = 0) es del 46\%, es decir, que el consumo de alcohol se lo reporta como inexistente. Ya en el análisis de los patrones de consumo, el 44\% presenta un consumo de tipo moderado; mientras que el $9.9 \%$ reporta la presencia de patrones de consumo de alcohol intenso.

Tabla 3.

Análisis comparativo por sexo del patrón de consumo de alcohol.

\begin{tabular}{|c|c|c|c|c|c|c|c|}
\hline \multirow{2}{*}{ VARIABLES } & \multicolumn{2}{|c|}{ Total } & \multicolumn{2}{|c|}{ Hombres } & \multicolumn{2}{|c|}{ Mujeres } & \multirow{2}{*}{$x^{2}(p)$} \\
\hline & $n$ & $\%$ & $n$ & $\%$ & $n$ & $\%$ & \\
\hline Abstinencia & 580 & $46.0 \%$ & 301 & $43.2 \%$ & 279 & $49.5 \%$ & \\
\hline Moderado & 555 & $44.1 \%$ & 334 & $47.9 \%$ & 221 & $39.3 \%$ & 7.74(.102) \\
\hline Intenso & 125 & $9.9 \%$ & 62 & $8.9 \%$ & 63 & $11.2 \%$ & \\
\hline
\end{tabular}

En el caso de los Episodios de consumo de alcohol, la tabla 4 muestra que de aquellos participantes que reportaron consumo (680 casos), el 82\% aproximadamente lo hace sin recurrir al consumo de más de cinco bebidas alcohólicas en un espacio corto de tiempo. El $11 \%$ reporta que ha tenido un ECIA alguna vez en el pasado reciente, el $6.6 \%$ al menos una al mes y el $0.3 \%$ de manera semanal.

Tabla 4.

Análisis comparativo por sexo del Patrón de consumo de alcohol

\begin{tabular}{lccccccc}
\hline \multirow{2}{*}{ VARIABLES } & \multicolumn{2}{c}{ Total } & \multicolumn{2}{c}{ Hombres } & \multicolumn{2}{c}{ Mujeres } & \multirow{2}{*}{$\boldsymbol{X}^{\mathbf{2}}$} \\
\cline { 2 - 7 } & $\boldsymbol{n}$ & $\boldsymbol{\%}$ & $\boldsymbol{N}$ & $\boldsymbol{\%}$ & $\boldsymbol{n}$ & $\boldsymbol{\%}$ & \\
\hline Sin episodios & 558 & $82.1 \%$ & 334 & $84.3 \%$ & 224 & $78.9 \%$ & \\
Una vez en el pasado reciente & 75 & $11.0 \%$ & 34 & $8.6 \%$ & 41 & $14.4 \%$ & \\
Al menos una mensual & 45 & $6.6 \%$ & 26 & $6.6 \%$ & 19 & $6.7 \%$ & \multirow{2}{*}{$6(.133)$} \\
Al menos una semanal & 2 & $0.3 \%$ & 2 & $0.5 \%$ & 0 & $0 \%$ & \\
\hline
\end{tabular}

Nota: Total: 680 casos; Hombres: 396; Mujeres: 284 ; $x^{2}$ : Chi cuadrado de Pearson; $p$ : significancia

En estos dos análisis no se evidenció la presencia significativa $(p>.05)$ de diferencias por sexo entre los participantes. Por lo que los patrones de consumo, así como la presencia de ECIA, no difieren entre los grupos y se consideran iguales. 


\section{Consumo de alcohol y grupos de edad}

Dado que el presente estudio cuenta con participantes con rangos de edad entre los 12 a los 19 años, una visualización (figura 1) de las medias obtenidas y agrupadas por la edad, muestra que, a medida que aumenta la edad, el consumo se incrementa, aunque no de manera continuada. En el mismo, se observa que entre los 12 a 15 años aumenta con cada año, para nivelarse en los grupos de 15 a los 18 años y generar un segundo proceso de incremento del consumo a partir de los 19 años.

También se observa que los grupos de 12 a 14 años tienen una media de consumo por debajo de la media global del estudio ( $M=3.8$ puntos), mientras que los grupos de 15 a 18 años se encuentran por encima de la media global del estudio. La media del consumo del grupo de 19 años o más ya supera el punto de corte para considerar que el consumo que mantienen ya es de riesgo.

Además, se establece la presencia de una correlación pequeña, positiva y significativa entre la edad y el consumo de alcohol registrado en el AUDIT.

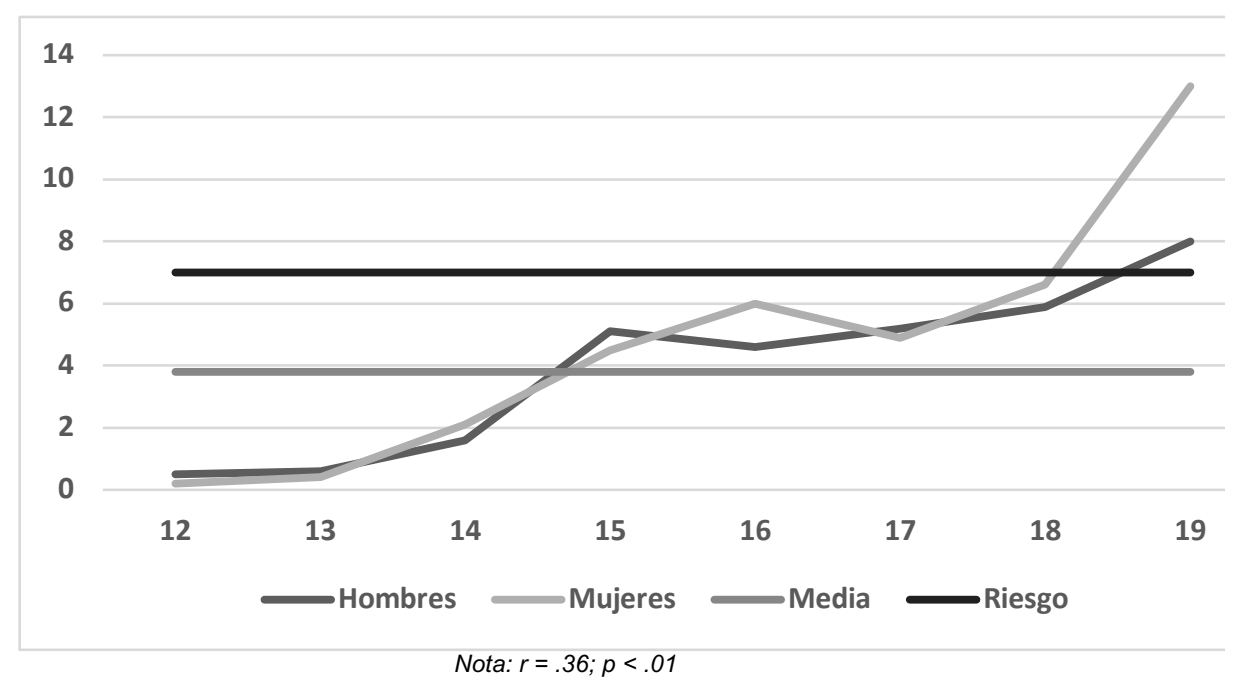

Figura 1. Distribución del consumo de alcohol por sexo según el grupo etario de pertenencia. Se comparan los resultados con la media global del estudio y el punto de corte referencial del AUDIT.

\section{DISCUSIÓN}

Los objetivos del presente estudio consistieron en identificar las diferencias de sexo existentes en torno al consumo de alcohol en una muestra de adolescentes del Ecuador, así como los niveles y los patrones de consumo existentes en una muestra de adolescentes ecuatorianos.

Con respecto al consumo general y sus consecuencias, los puntajes se encuentran por debajo del punto de corte, por lo que el consumo como tal es bajo y sin riesgo significativo. Estos hallazgos en el Ecuador coinciden con estudios preliminares actuales (Moreta-Herrera et al., 2018a; MayorgaLascano et al., 2019). Sin embargo, de estudios anteriores se aprecia que el consumo con el paso del tiempo ha ido aumentando (Consejo Nacional de Control de Sustancias Estupefacientes y Psicotrópicas, Observatorio Nacional de Drogas, 2013). Esta impresión muestra que la dinámica del consumo muy probablemente es inicial entre los adolescentes y sin una forma definida. Aparentemente, el consumo que caracteriza a este grupo no representa riesgo 
(determinado por el punto de corte). No obstante, dado que se trata de adolescentes, se debe considerar que, usando otros puntos de corte propuestos, es probable que esta impresión de ausencia de riesgo cambie (Galán, González, \& Valencia-Martín, 2014). En este aspecto, el estudio se limita a reportar los resultados según los parámetros de mayor aceptación, debido a que no hay un consenso pleno sobre estos estimadores en adolescentes.

Sobre las categorias del consumo, mayoritariamente los participantes tienen un consumo o efectos del consumo de alcohol sin riesgo (76\%), seguido por un consumo de tipo riesgoso (22\%) y un $2 \%$ aproximadamente con problemas físicos, psicosociales y/o probable dependencia. Estos hallazgos no pueden ser contrastados con estudios internacionales referenciales debido a la falta de sistematización. Sin embargo, en el caso del Ecuador, los hallazgos señalan que esta situación no ha cambiado y se sostiene (Paqui Baho, 2017).

En cuanto a los patrones de consumo formulados, se encuentra que el $46 \%$ de los participantes son abstemios, pues no reportan tener consumo alguno. Un 44\% aproximadamente manifiesta tener un consumo leve y/o moderado de baja frecuencia, cantidad y concentración alcohólica. Mientras que el $9 \%$ reporta consumos más intensos en frecuencia y cantidad de bebidas y concentración alcohólica.

Al analizar la presencia del consumo intenso de alcohol, el $11 \%$ reportó un ECIA al menos una vez en el pasado reciente, $6.6 \%$ al menos uno mensual y $0.3 \%$ al menos uno semanal. Los reportes de ECIA en la muestra ecuatoriana difieren de otros estudios locales realizados en España y México (Golpe et al., 2017; Villarreal-González et al., 2013). Mientras que en estudios ecuatorianos resultan ser mayores (Ramón Durán \& Dávila Pontón, 2018). Los ECIA se vuelven más recurrentes en la adolescencia media y tardia que en la adolescencia inicial (Miller et al., 2016), por lo que se evidencia el factor relevante de la edad con respecto al consumo.

Por tanto, los datos llevan a considerar que el consumo de alcohol entre los adolescentes es bajo al tomar como referencia paises de la esfera europea y norteamericana (SAMHSA, 2013; Valencia-Martín et al., 2007; Zurita \& Álvaro, 2014) al igual que en países de la región como México, Chile y Argentina (Arrioja-Morales et al., 2017; Morello et al., 2017; Servicio Nacional para la Prevención y Rehabilitación del consumo de drogas y alcohol, 2013), en el que resulta ser más bajo. Estos hallazgos reflejan que, al menos en la etapa de la adolescencia, el consumo de los ecuatorianos tiene mayor similitud al de países mediterráneos que al de la región norte de Europa (Heath, 1997; Stranges et al., 2006).

Acerca de la edad y el consumo, este se presenta de manera temprana (12 años en adelante), aunque en una porción pequeña, que se reparte a lo largo de la adolescencia. Los resultados concuerdan con los estudios en EUA (Center for Behavioral Health Statistics and Quality, 2018), al señalar que el inicio y sostenimiento del consumo de alcohol empieza en la adolescencia temprana. Se confirma la relación positiva entre la edad y el consumo de alcohol (Kunstsche et al., 2015; Liang \& Chikritzhs, 2015; Miller et al., 2016) y la manera en cómo se consolida la habituación y la problemática a futuro (Gmel et al., 2011; León-Muñoz et al., 2017).

Además, la franja comprendida entre los 15 a los 18 años corresponde a las edades en que el consumo se consolida como una práctica habitual, aunque no de riesgo. Este momento es crítico, porque en esta etapa se afianza 
el consumo y la combinación con otras sustancias (Zurita \& Álvaro, 2014). Si describimos la transición de la adolescencia, la inicial corresponde al inicio y la experimentación, la media representa la consolidación del hábito en niveles no riesgosos y la tardía e inicio de la juventud, la etapa para la transición hacia el consumo de riesgo. No se hallaron estudios referenciales que establezcan estos criterios desde una mirada longitudinal.

Finalmente, el aspecto de sexo despierta interés, pues en todos los resultados de este trabajo no se apreciaron diferencias significativas, lo que difiere de trabajos previos (Arrioja-Morales et al., 2017; Miller et al., 2016; Villarreal-González et al., 2013; World Health Organization, 2014). Así, aunque se requieren más estudios a fin de confirmar o no estos resultados encontrados, se puede evidenciar que el consumo por parte del sexo femenino está cambiando y direccionándose a un incremento cada vez mayor, y es muy probable que en adelante la brecha entre hombres y mujeres disminuya o desaparezca (Wilsnack et al., 2013).

Sobre las implicaciones del estudio, se encuentra una práctica generalizada del consumo con baja abstinencia entre los adolescentes y sin diferencias por sexo. Aunque no es intenso y al ser moderado, muestra ciertas características similares al de otras regiones geográficas (el mediterráneo), con una proporción pequeña de casos con niveles intensos que desencadenan problemáticas o necesidades de observación e intervención específica.

Las implicaciones prácticas ayudarán al desarrollo de procesos de intervención orientados a la prevención educativa sobre la base de evidencia de primera mano. Su implementación no requerirá de adaptaciones significativas y puede representar ahorro y eficiencia a nivel de costos en servicios de salud y formación profesional. De igual manera, permitirá tomar correctivos a nivel educativo y de intervención primaria en la prevención del consumo, el riesgo y el daño real.

\section{Limitaciones y recomendaciones a futuro}

Una limitación considerable es la dificultad para homologar distintos estudios nacionales y regionales por la variedad de metodologias e intereses que convergen sobre el tema. Como se señala anteriormente, gran parte de la dificultad se centra en categorizar adecuadamente el problema del consumo a partir del tipo y forma de ingesta del alcohol (Kuntsche et al., 2015), junto con la cantidad correcta, pues tiende a variar en distintas legislaciones (World Health Organization, 1992).

\section{REFERENCIAS}

Arrioja Morales, G., Frari Galera, S., Gargantúa Aguila, S., de Ávila Arroyo, M., \& Morales Castillo, F. (2017). Factores de riesgo para el consumo de alcohol en adolescentes. Revista Eletrônica Saúde Mental Álcool e Drogas, 13(1), 22-29. https://doi.org/10.11606/issn.1806-6976.v13i1p22-29

Ato, M., López, J., \& Benavente, A. (2013). Un sistema de clasificación de los diseños de investigación en psicologia. Anales de Psicología, 29(3), 1038-1059. https://doi.org/10.6018/analesps.29.3.178511

Boubeta, A. R., Ferreiro, S. G., Gallego, M. A., Tobío, T. B., \& Mallou, J. V. (2017). Validación del "Test de Identificación de Trastornos por Consumo de Alcohol" (AUDIT) en población adolescente española. Psicología Conductual, 25(2), 371.

Center for Behavioral Health Statistics and Quality (2018). 2017 National Survey on Drug Use and Health: Detailed tables. Rockville, MD: Substance Abuse and Mental Health Service Administration.

Cerdá, M., Diez-Roux, A. V., Tchetgen Tchetgen, E., Gordon-Larsen, P., \& Kiefe, C. (2010). The Relationship Between Neighborhood Poverty and Alcohol Use: Estimation by Marginal Structural Models. Epidemiology, 21(4), 482-489. https://doi.org/10.1097/EDE.0b013e3181e13539

Consejo Nacional de Control de Sustancias Estupefacientes y Psicotrópicas, Observatorio Nacional de Drogas (2013). Cuarta encuesta nacional sobre uso de drogas en estudiantes de 12 a 17 años. Quito: Imprenta Full Color. 
de la Villa Moral Jiménez, M., Bringas Molleda, C., Ovejero Bernal, A., Morales Quintero, L. A., \& Rodriguez Diaz, F. J. (2017). Emergencia sociosanitaria en consumo de riesgo de alcohol y sintomas de dependencia en jóvenes. Salud y drogas, 17(2), 91-99. https://doi.org/10.21134/haaj.v17i2.307

Gaete, J., Olivares, E., Rojas-Barahona, C. A., Rengifo, M. J., Labbé, N., Lepe, L., . . . Chen, M. Y. (2016). Consumo de tabaco y alcohol en adolescentes de 10 a 14 años de la ciudad de San Felipe, Chile: prevalencia y factores asociados. Revista médica de Chile, 144(4), 465-475. https: / / doi.org/ 10.4067/S0034-98872016000400007

Galán, I., González, M., \& Valencia-Martín, J. L. (2014). Patrones de consumo de alcohol en España: un país en transición. Revista Española de Salud Pública, 88(4), 529-540. https://doi.org/10.4321/S113557272014000400007

Gea, A., Bes-Rastrollo, M., Toledo, E., Garcia-Lopez, M., Beunza, J. J., Estruch, R., \& Martinez-Gonzalez, M. A. (2014). Mediterranean alcohol-drinking pattern and mortality in the SUN (Seguimiento Universidad de Navarra) Project: a prospective cohort study. British Journal of nutrition, 111(10), 1871-1880. https://doi.org/10.1017/S0007114513004376

Gmel, G., Kuntsche, E., \& Rehm, J. (2011). Risky single-occasion drinking: bingeing is not bingeing. Addiction, 106(6), 1037-1045. https://doi.org/10.1111/j.1360-0443.2010.03167.x

Golpe, S., Isorna, M., Barreiro, C., Braña, T., \& Rial, A. (2017). Consumo intensivo de alcohol en adolescentes: prevalencia, conductas de riesgo y variables asociadas. Adicciones, 29(4), $256-267$. https://doi.org/10.20882/adicciones.932

Heath, D. (1997). Cultural variations among drinking patterns. En M. Grant, \& J. Litvak (Edits.), In Drinking Patterns and their Consequences (págs. 103-128). Washington, DC: Taylor \& Francis.

Kuntsche, E., Wicki, M., Windlin, B., Roberts, C., Gabhainn, S. N., Van Der Sluijs, W., . . . Tynjälä, J. (2015). Drinking motives mediate cultural differences but not gender differences in adolescent alcohol use. Journal of Adolescent Health, 56(3), 323-329. https://doi.org/10.1016/j.jadohealth.2014.10.267

León-Muñoz, L. M., Guallar-Castillón, P., García-Esquinas, E., Galán, I., \& Rodríguez-Artalejo, F. (2017). Alcohol drinking patterns and risk of functional limitations in two cohorts of older adults. Clinical nutrition, 36(3), 831-838. https://doi.org/10.1016/j.clnu.2016.05.005

Liang, W. \& Chikritzhs, T. (2015). Age at first use of alcohol predicts the risk of heavy alcohol use in early adulthood: A longitudinal study in the United States. International Journal of Drug Policy, 26(2), 131134. https://doi.org/10.1016/j.drugpo.2014.07.001

Maldonado-Molina, M., Reingle, J., \& Jennings, W. (2011). Does alcohol use predict violent behaviors? The relationship between alcohol use and violence in a nationally representative longitudinal sample. Youth Violence and Juvenile Justice, 9, 99-111. https://doi.org/10.1177/1541204010384492

Mayorga-Lascano, M., Moreta-Herrera, R., León-Tamayo, L., \& Troncozo-Guevara, M. (2019). Actitudes favorables y consumo de alcohol en adolescentes ecuatorianos. Análisis correlacional y comparativo entre grupos de riesgo y no riesgo. Health \& addictions/Salud y drogas, 19(2), 139-138.

Miller, P. G., Butler, E., Richardson, B., Staiger, P. K., Youssef, G. J., Macdonald, J. A., . . . Olsson, C. A. (2016). Relationships between problematic alcohol consumption and delinquent behaviour from adolescence to young adulthood. Drug and alcohol review, 35(3), 317-325. https://doi.org/10.1111/dar.12345

Morello, P., Pérez, A., Peña, L., Braun, S. N., Kollath-Cattano, C., Thrasher, J. F., . . . Mejía, R. (2017). Factores de riesgo asociados al consumo de tabaco, alcohol y otras drogas en adolescentes escolarizados de tres ciudades de Argentina. Archivos argentinos de pediatria, 115(2), $155-159$. https://doi.org/10.5546/aap.2017.155

Moreta-Herrera, R., Ilaja-Verdesoto, B., Mayorga-Lascano, M., León-Tamayo, L., \& López-Castro, J. (2018a). Actitudes y disposición personal ante el consumo y exposición a sustancias en adolescentes del Ecuador. Salud y Drogas, 18(2), 217-226. https://doi.org/10.21134/haaj.v18i2.383

Moreta-Herrera, R., Mayorga-Lascano, P., León-Tamayo, A., \& Ilaja-Verdesoto, B. (2018b). Consumo de sustancias legales, ilegales y fármacos en adolescentes y factores de riesgo asociados a la exposición reciente. Salud y drogas, 18(1), 39-50. https://doi.org/10.21134/haaj.v18i1.333

Navarro-Oliva, E. I., Robledo, P. J., Medina, M. E., Candía-Arredondo, J. S., \& Facundo, F. R. (2016). Exposición a contenidos de uso de alcohol en Facebook y consumo de alcohol en adolescentes. Revista de enfermería Herediana, 9(1), 43-49. https: / / doi.org/ 10.20453/renh.v9i1.2863

Observatorio Español de las drogas y las adicciones (2018). Informe 2018. Alcohol, tabaco y drogas ilegales en España. Madrid: Ministerio de Sanidad, Consumo y Bienestar Social.

Paqui Baho, K. (2017). Funcionalidad familiar y su relación con el consumo de alcohol en adolescentes de Bachillerato de la Zona 7 Ecuador (master's thesis). Loja: Universidad Nacional de Loja.

Pérez, C. L. \& Lara, C. V. (2008). Asertividad, resistencia a la presión de grupo y consumo de alcohol en universitarios. Acta colombiana de psicologia, 11(1), 155-162.

$\mathrm{R}$ Core Team. (2015). R: A language and environment for statistical computing. Vienna, Austria: R Foundation for Statistical Computing.

Ramón Durán, K. \& Dávila Pontón, Y. (2018). Consumo de alcohol en adolescentes de bachillerato Estudio en el Colegio César Dávila Andrade (trabajo de titulación de pregrado). Cuenca: Universidad de Cuenca.

Rehm, J., Mathers, C., Popova, S., Thavorncharoensap, M., Teerawattananon, Y., \& Patra, J. (2009). Global burden of disease and injury andeconomic cost attributable to alcohol use and alcohol-use disorders. The Lancet, 2223-2233. https://doi.org/10.1016/S0140-6736(09)60746-7

Rubio, G., Bermejo, J., Cabellero, M., \& Santo Domingo, J. (1998). Validación de la prueba para la identificación de trastornos por uso de alcohol (AUDIT) en atención primaria. Revista Clínica Española, 198, 11-14.

SAMHSA. (2014). Results from the 2013 National Survey on Drug Use and Health: Summary of National Findings. Rockville, MD: NSDUH Series H-48. 
Saunders, J., Aasland, O., Babor, T., De la Fuente, J., \& Grant, M. (1993). Development of the Alcohol Use Disorders Identification Test (AUDIT): WHO collaborative project on early detection of persons with harmful alcohol consumption-II. Addiction, 88, 791-804. https://doi.org/10.1111/j.13600443.1993.tb02093.x

Schulte, M. T., Ramo, D., \& Brown, S. A. (2009). Gender differences in factors influencing alcohol use and drinking progression among adolescents. Clinical psychology review, 29(6), 535-547. https://doi.org/10.1016/j.cpr.2009.06.003

Servicio Nacional para la Prevención y Rehabilitación del consumo de drogas y alcohol (2013). Décimo Estudio Nacional de Drogas en Población Escolar de Chile, $8^{\circ}$ básico a $4^{\circ}$ medio. Santiago de Chile: SENDA - Observatorio Chileno de Drogas. https://doi.org/10.5354/0717-3652.2004.20213

Stranges, S., Notaro, J., Freudenheim, J. L., Calogero, R. M., Muti, P., Farinaro, E., . . Trevisan, M. (2006). Alcohol drinking pattern and subjective health in a population-based study. Addiction, 101(9), 12651276. https://doi.org/10.1111/j.1360-0443.2006.01517.x

Valencia-Martín, J. L., Galán, I., \& Rodríguez-Artalejo, F. (2007). Binge drinking in Madrid, Spain. Alcoholism: Clinical and Experimental Research, 31(10), 1723-1730. https://doi.org/10.1111/j.15300277.2007.00473.x

Vieno, A., Altoè, G., Kuntsche, E., \& Elgar, F. J. (2018). Do public expenditures on health and families relate to alcohol abstaining in adolescents? Multilevel study of adolescents in 24 countries. Drug and alcohol review, 37, S120-S128. https://doi.org/10.1111/dar.12696

Villarreal González, M. E., Sánchez Sosa, J. C., \& Musitu Ochoa, G. (2013). Análisis psicosocial del consumo de alcohol en adolescentes mexicanos. Universitas Psychologica, 12(3), 857-873. https://doi.org/10.11144/Javeriana.UPSY12-3.apca

Wadolowski, M., Hutchinson, D., Bruno, R., Aiken, A., Clare, P., Slade, T., . . . Mattick, R. P. (2015). Early adolescent alcohol use: are sipping and drinking distinct? Alcoholism: clinical and experimental research, 39(9), 1805-1813. https://doi.org/10.1111/acer.12826

Wesche, R., Kreager, D. A., \& Lefkowitz, E. S. (2018). Sources of Social Influence on Adolescents' Alcohol Use. Journal of Research on Adolescence, 29(4), 984-1000. https://doi.org/10.1111/jora.12439

Wilsnack, S. C., Wilsnack, R. W., \& Kantor, L. W. (2013). Focus on: Women and the costs of alcohol use. Alcohol Research: Current Reviews, 35(2), 219-228.

World Health Organization (1992). AUDIT. The Alcohol Use Disorders Identification Test: guidelines for use in primary health care. Geneva: WHO.

World Health Organization (1994). Lexicon of alcohol and drug terms. Geneve: WHO.

World Health Organization (2013). WHO Report on Global Tobacco Epidemic, 2013. Enforcing bans on tobacco advertising promotion and sponsorship. Luxemburg: World Health Organization.

World Health Organization (2014). Global Status Report on Alcohol and Health. Geneva: WHO.

Zurita, F. \& Álvaro, J. I. (2014). Repercusión del tabaco y alcohol sobre factores académicos y familiares en adolescentes. Health and Addictions 14(1), 59-70. https://doi.org/10.21134/haaj.v14i1.214

Recibido 06-05-2019 | Aceptado 20-09-2019

Este trabajo se encuentra bajo una Licencia Creative Commons Atribución 4.0 Internacional que permite a terceros utilizar lo publicado siempre que se dé el crédito pertinente a los autores y a Psicodebate 